\title{
Endoscopías digestivas altas y biopsias gástricas en la Clínica Médica Cayetano Heredia.
}

Gastrointestinal endoscopies and gastric biopsies at Clinica Medica Cayetano Heredia

TORRES SILVA Elder***, CABELLO José*, SALINAS César**, COK Jaime**, BUSSALLEU Alejandro*.

* Departamento de Medicina. Universidad Peruana Cayetano Heredia.

** Departamento de Patología. Universidad Peruana Cayetano Heredia.

*** Graduando de Medicina Humana. Universidad Peruana Cayetano Heredia.

\section{SUMMARY}

Objective: To establish the endoscopic and histologic diagnosis, including Helicobacter pylori infection, of patients with dyspepsia, and to determine the relation between endoscopic and histologic findings. Material and Methods: Upper endoscopies done in 435 patients at the Clinical Médica Cayetano Heredia from September $1^{\text {st }} 1993$ to January $31^{\text {st }} 1996$ were reviewed. Results: Gastric biopsies were performed in 48.9\% of all the patients. Gastritis was diagnosed by endoscopyc means in $56.78 \%$ and histologically in $\mathbf{9 1 . 0 5 \%}$. Helicobacter pylori was diagnosed in $\mathbf{7 8 . 6 9 \%}$. A significant association was found between Helicobacter pylori and active gastritis, mucin injury and gastric ulcer. (Rev Med Hered 1997; 8: 58-66).

KEY WORD: Upper endoscopy, gastric biopsies, gastritis, Helicobacter pylori.

\section{RESUMEN}

Objetivo: Determinar los diagnósticos endoscopios e histopatológicos de las biopsias gástricas en pacientes sometidos a endoscopía digestiva alta, la frecuencia de Helicobacter pylori en biopsias gástricas no neoplásicas y su relación con el diagnóstico endoscópico e histopatológico. Material y métodos: Estudio retrospectivo de 435 endoscopías realizadas en la Clínica Médica Cayetano Heredia entre el $1^{\circ}$ de setiembre de 1993 y el 31 de enero de 1996. Se realizó la relectura de 104 láminas por un mismo examinados, para estandarizar los informes finales de anatomía patológica. Resultados: La edad promedio fue de 44.41 
años, el $47.36 \%$ fueron varones y el 52.64\%, mujeres. La gastritis fue el diagnóstico endoscópico mas frecuente (56.78\%). Asimismo, gastritis fue el diagnóstico histológico mas frecuente $(91.05 \%)$, de la cuales fueron gastritis activas $91.9 \%$, con daño mucinoso $98.8 \%$ y con metaplasia intestinal $22.5 \%$. En el $48.97 \%$ de la población estudiada se realizó biopsia endoscópica. La frecuencia de Helicobacter pylori en mucosa gástrica no neoplásica fue de $78.69 \%$, y se encontró asociación estadísticamente significativa entre la presencia de Helicobacter pylori y hallazgo histológico de gastritis, actividad de la gastritis, presencia de daño mucinoso y el diagnóstico endoscópico de úlcera gástrica. (Rev Med Hered 1997; 8:58-66).

PALABRAS CLAVE: Endoscopia digestiva alta, gastritis, Helicobacter pylori, biopsia gástrica.

\section{INTRODUCCION}

La práctica endoscópica en el Perú siempre ha tratado de ponerse al nivel de los avances de la tecnología de su época; se dispone de información que el Dr. Alfredo Calderón Martínez realiza las primeras endoscopías con un gastroscopio rígido de Schindler y poco después el Dr. Jorge Diez Canseco, impulsor de la especialidad en el Hospital Dos de Mayo. Posteriormente en 1942, en el Hospital Arzobispo Loayza, se realizan gastroscopías con un instrumento semiflexible (tubo metálico con un resorte terminal y todo rodeado con una cubierta de caucho) de visión lateral, éstas eran practicadas por el Dr. Ricardo Sáenz Jiménez y ayudado por el entonces alumno del 5to año de medicina Germán Garrido Klinge. En la década de los cincuentas, en diversos centros hospitalarios de Lima, se realizan endoscopías con instrumentos semiflexibles de diversos modelos. En 1962 llega al Perú y en particular al Hospital Edgardo Rebagliatti Martins el primer fibroscopio modelo Hirschowitz Americano sin ángulo regulable; con este instrumento realiza las primeras duodenoscopías el Dr. Hernán Espejo Romero. Los modelos de fibroscopios, desde ese entonces, fueron sofisticándose y poco a poco fueron adquiriéndose en diversos centros hospitalarios de Lima. En 1974 se realizan las primeras colangiografías retrógradas endoscópicas. Posteriormente, como en cualquier otro centro del mundo, se han ido realizando, a través del endoscopio, la extracción de pólipos (1), el tratamiento de úlceras sangrantes, dilataciones, gastrostomías, escleroterapia y ligadura de várices esofágicas, colangiografías retrógradas, etc. En 1995 se empieza a realizar las primeras ultrasonografías endoscópicas. Como vemos el uso que se le ha dado a la endoscopía digestiva alta, se ha ido incrementado con el tiempo. Hay mucha literatura nacional e internacional de trabajos realizados en base a endoscopías digestivas altas; los temas son muy variados debido a la gran patología que existe a nivel de esófago, estómago y duodeno.

El presente es un estudio retrospectivo, que describe los resultados de las endoscopías digestivas altas y de las biopsias gástricas, realizadas en pacientes de la Clínica Médica Cayetano Heredia (CLIMA), con el objetivo de:

Determinar los diagnósticos endoscopios e histopatológicos de las biopsias gástricas. Determinar en qué tipo de diagnóstico endoscópico se realizaron biopsias gástricas. 
Determinar la frecuencia del Helicobacter pylori en las biopsias gástricas no neoplásicas. Relacionar el diagnóstico endoscópico con el diagnóstico histológico de las biopsias gástricas no neoplásicas.

a) Relacionar la presencia del Helicobacter pylori con el diagnóstico endoscópico y con el hallazgo histológico en las biopsias gástricas no neoplásicas.

\section{MATERIALES Y METODOS}

Se realizó una evaluación retrospectiva de todos los pacientes a los que se les realizó esófago-gastro-duodenoscopía por diversas molestias digestivas altas, fundamentalmente dispepsia, pertenecientes a la CLIMA entre el $1^{\text {ro }}$ de setiembre de 1993 y el 31 de enero de 1996.

La recolección de la información relacionada a los diagnósticos endoscopios se obtuvo del archivo de endoscopías digestivas altas del Servicio de Gastroenterología de la CLIMA. Para simplificar el reporte de los resultados y facilitar el trabajo estadístico, en aquellos casos en que se encontraron varios diagnósticos endoscopios se consideró el que aparentemente tenía mayor importancia en relación al cuadro clínico que presentaba el paciente.

Se realizó además la recolección de los informe de histopatología de todas las biopsias gástricas obtenidas a través de la endoscopia en el Servicio de Inmunopatología.

Se realizó la relectura de 104 láminas por un mismo examinador, para estandarizar los informes finales de anatomía patológica.

En la consideración de los hallazgos histológicos de gastritis y de Helicobacter pylori, se tomaron en cuenta sólo los reportes de mucosa gástrica no neoplásica.

\section{Definición de términos:}

a)Gastritis: Es la inflamación de la mucosa gástrica, hay mucha controversia en su clasificación y esta se realiza según criterios endoscopios e histológicos $(5,16,20,46)$ :

Endoscópicamente se clasifica en:

Gastritis aguda: caracterizada por hemorragias subepiteliales, petequias, erosiones, con mucosa friable y sangrante; estas lesiones son superficiales, y pueden variar en número, tamaño y localización.

Gastritis crónica: caracterizado por eritema de la mucosa gástrica, engrosamiento de pliegues, aplanamiento de pliegues, palidez de la mucosa, adelgazamiento de la mucosa con visualización de vasos submucosos; y pueden variar en su extensión y localización. Histológicamente se ha seguido la clasificación utilizada en el Servicio de Patología del Hospital Nacional Cayetano Heredia (5) la que considera: 
Gastritis crónica superficial: hay un infiltrado de células infiltrado en la porción superficial de la mucosa, que llega hasta el cuello de las glándulas propias (foveola), en particular en la lámina propia entre las criptas gástricas.

Gastritis crónica profunda: en la que el infiltrado de células inflamatorias pasa la zona de la foveola y llega a las porciones profundas de la mucosa.

Gastritis crónica atrófica: hay destrucción y desaparición de las glándulas, independientemente del grado de inflamación, dejando solamente pequeños hoyos.

La actividad de la gastritis está dada por la presencia de células inflamatorias agudas (polimofonucleares) y se la clasifica en leve, moderada y severa.

b) Metaplasia: Es el cambio de las células de la superficie y del epitelio de las criptas gástricas por células de morfología similar a células del intestino; se clasifica en: Completa (Tipo I) similares a células del intestino delgado, e incompleta (Tipo II) similares a células del intestino grueso; puede haber en una misma muestra una combinación de los dos tipos de metaplasia que se denomina tipo mixta $(5,46)$.

c) Displasia: definida por alteración en la proliferación celular, pérdida de la diferenciación citoplasmática de las mismas, atipia y pérdida de la arquitectura de la mucosa. Se le considera como una lesión precancerosa.

d) Daño mucinoso, que está definido como la depleción de la vacuola de mucina de las células de la foveola gástrica; ésta a su vez se clasifica en (5):

Parcial: En donde la depleción es incompleta de la vacuola de mucina de las células del a faveola gástrica.

Total: Es la depleción completa de la vacuola de mucina de las células foveolares gástricas.

Focal: En el que sólo se aprecia el daño en una zona del epitelio; ésta a su vez puede ser parcial o total.

Multifocal: En el que el daño se aprecia en varias zonas del epitelio gástrico; igualmente puede ser parcial o total.

Difusa: En el que se aprecia una depleción amplia y extendida de la vacuola de mucina del epitelio foveolar; también puede ser parcial o total.

e) Helicobacter pylori: se trata de una bacteria espiralaza, gram negativa, microaerófila, ureasa positiva, cuyo hábitat es el epitelio gástrico y que se le diagnóstica por diversos métodos, siendo el usado en este trabajo la coloración de hematoxilina.

\section{RESULTADOS}


Se revisaron en total 435 esofagogastroduodenoscopías de la CLIMA, en las cuales se obtuvieron los siguientes resultados:

\section{Edad y Sexo}

La edad promedio fue de 44.41 años, con edades entre los 4 y 94 años, sin predominio de un grupo de edad. La edad promedio entre ambos sexos fue similar. Hubo 206 hombres $(47.36 \%)$ y 229 mujeres $(52.64 \%)$.

\section{Diagnósticos endoscópicos}

El resultado de los hallazgos endoscopios se encuentran en la tabla $\mathrm{N}^{\mathrm{o}} 1$.

\begin{tabular}{|lrc|}
\hline Tabla $\mathbf{N}^{\circ} 1$. Diagnósticos endoscópicos. \\
Diagnóstico & $\mathrm{n}$ & \multicolumn{1}{c|}{$\%$} \\
\hline Normal & 60 & 13.79 \\
Gastritis & 247 & 56.78 \\
Esofagitis & 56 & 12.87 \\
Ulcera péptica & 28 & 6.44 \\
$\quad$ - Ulcera duodenal & 17 & 3.91 \\
$\quad$ - Ulcera gástrica & 11 & 2.53 \\
Pólipo gástrico & 14 & 3.22 \\
Cicatriz de úlcera duodenal & 10 & 2.30 \\
Cáncer & 7 & 1.61 \\
Várices esofágicas & 5 & 1.15 \\
Duodenitis & 4 & 0.92 \\
Cicatriz de úlcera gástrica & 2 & 0.46 \\
Pólipo esofágico & 1 & 0.23 \\
Pólipo duodenal & 1 & 0.23 \\
TOTAL & & \\
\hline
\end{tabular}

Realización de biopsias endoscópicas

Se realizaron en total 213 biopsias endoscópicas las que representan el $48.97 \%$ del total de endoscopías. La realización de biopsias por diagnósticos endoscopios se encuentran en la tabla $\mathrm{N}^{\mathrm{o}} 2$. 


\begin{tabular}{|c|c|c|c|c|c|}
\hline \multirow[t]{3}{*}{ Tabla $\mathrm{N}^{\circ}$ 2. $\mathrm{Re}$} & \multicolumn{5}{|c|}{$\begin{array}{l}\text { Relación entre diagnóstico endoscópico y } \\
\text { realización de biopsia. }\end{array}$} \\
\hline & \multicolumn{2}{|c|}{ BIOPSIA } & \multicolumn{2}{|c|}{ NO BIOPSIA } & \multirow[b]{2}{*}{ TOTAL } \\
\hline & $n$ & $\%$ & $n$ & $\%$ & \\
\hline Cáncer & 7 & 100 & 0 & 0.00 & 7 \\
\hline Ulcera gástrica & 10 & 90.91 & 1 & 9.09 & 11 \\
\hline Polipos & 14 & 87.50 & 2 & 12.50 & 16 \\
\hline Ulcera duodenal & 11 & 64.71 & 6 & 35.29 & 17 \\
\hline Gastritis & 139 & 56.28 & 108 & 43.72 & 247 \\
\hline Cicatriz de úlcera & 6 & 50.00 & 6 & 50.00 & 12 \\
\hline Esolagitis & 23 & 41.07 & 33 & 58.93 & 56 \\
\hline Várices esolágicas & 1 & 20.00 & 4 & 80.00 & 5 \\
\hline NORMAL & 2 & 3.33 & 58 & 96.67 & 60 \\
\hline Duodenitis & 0 & 0.00 & 4 & 100 & 4 \\
\hline TOTAL & 213 & 48.97 & 222 & 51.03 & 435 \\
\hline
\end{tabular}

\section{Hallazgos histológicos en las biopsias gástricas.}

Encontramos que se reportan mucosas gástricas histológicamente como normales en el $5.6 \%$ de las biopsias; como cáncer gástrico en 3.68\% de las biopsias; y con algún tipo de gastritis en el 91.05\%; de éstas fueron activas en el 91.91\%; presentaron algún tipo de daño mucinoso en el 98.84\%; y algún tipo de metaplasia intestinal en el $22.54 \%$ de las gastritis. La frecuencia de Helicobacter pylori en las muestras de mucosa gástrica no neoplásica es de $78.69 \%$.

Relación entre Helicobacter pylori en la mucosa gástrica no neoplásica y sexo.

No hay diferencia entre ambos sexos respecto a la presencia de Helicobacter pylori en las mucosas gástricas no neoplásicas.

Relación entre Helicobacter pylori y hallazgo histológico de gastritis en la mucosa gástrica no histológica de gastritis en la mucosa gástrica no neoplásica.

Se encontró una asociación estadísticamente significativa entre la presencia de Helicobacter pylori y el diagnóstico histológico de gastritis (Tabla №3). 


\begin{tabular}{|c|c|c|}
\hline \multicolumn{3}{|c|}{$\begin{array}{l}\text { Tabla N³. Helicobacter pylori (HP) y hallazgo histológico } \\
\text { de gastritis en la mucosa gástrica no neoplásica. }\end{array}$} \\
\hline $\begin{array}{l}\text { PRESENCIA DE } \\
\mathrm{HP}\end{array}$ & $\begin{array}{c}\text { GASTRITIS } \\
n(\%)\end{array}$ & $\begin{array}{c}\text { NORMAL } \\
n(\%)\end{array}$ \\
\hline $\mathrm{HP}(+)$ & $141(81.5)$ & $3(30)$ \\
\hline $\mathrm{HP}(-)$ & $32(18.5)$ & $7(70)$ \\
\hline TOTAL & 173 & 10 \\
\hline \multicolumn{3}{|l|}{$\begin{array}{l}P<0.0009 \\
\text { OR: } 10.28\end{array}$} \\
\hline
\end{tabular}

Relación entre Helicobacter pylori y actividad de gastritis en la mucosa gástrica no neoplásica.

Hubo asociación estadísticamente significativa entre la presencia de Helicobacter pylori y la actividad de la gastritis (Tabla $\mathrm{N}^{\circ} 4$ ).

\begin{tabular}{|c|c|c|}
\hline \multicolumn{3}{|c|}{$\begin{array}{c}\text { Tabla }{ }^{\circ} 4 \text {. Helicobacter pylori (HP) y actividad de } \\
\text { gastritis en la mucosa gástrica no neoplásica. }\end{array}$} \\
\hline PRESENCIA DE HP & $\begin{array}{l}\text { ACTIVA } \\
n(\%)\end{array}$ & $\begin{array}{l}\text { NO ACTIVA } \\
n(\%)\end{array}$ \\
\hline$H P(+)$ & $138(86.79)$ & $3(21.43)$ \\
\hline$H P(-)$ & $21(13.21)$ & $11(78.57)$ \\
\hline TOTAL & 159 & 14 \\
\hline $\begin{array}{l}P<0.0000004 \\
\text { OR: } 24.10\end{array}$ & & \\
\hline
\end{tabular}

Relación entre Helicobacter pylori y daño mucinoso en la mucosa gástrica no neoplásica.

Se encontró relación significativa entre la presencia de Helicobacter pylori y el daño mucinoso de cualquier tipo (Tabla $\mathrm{N}^{\circ} 5$ ). 


\begin{tabular}{|lcc|}
\hline $\begin{array}{c}\text { Tabla No5. Helicobacter pylori (HP) y daño mucinoso } \\
\text { en la mucosa gástrica no neoplásica. }\end{array}$ \\
\hline $\begin{array}{lcc}\text { PRESENCIA } & \text { DAÑO MUCINOSO } \\
\text { DE HP } & n(\%) & \text { NO DAÑO MUCINOSO } \\
\text { n (\%) }\end{array}$ \\
\hline HP (+) & $143(83.63)$ & $0(0.00)$ \\
HP $(-)$ & $28(16.37)$ & $2(100.0)$ \\
TOTAL & 171 & 2 \\
\hline P 0.0018 & & \\
OR: no definido & & \\
\end{tabular}

Relación entre el diagnóstico endoscópico y la presencia de Helicobacter pylori en la mucosa gástrica no neoplásica.

Comparado con el diagnóstico endoscópico de normalidad observamos que hay relación estadísticamente significativa entre el diagnóstico de úlcera gástrica y la presencia de Helicobacter pylori (Tabla $\mathrm{N}^{\mathrm{0}} 6$ ).

\begin{tabular}{|c|c|c|c|c|}
\hline \multicolumn{5}{|c|}{$\begin{array}{c}\text { Tabla N6. Diagnóstico endoscópico y presencia de Helicobacter pylori (HP) } \\
\text { en la mucosa gástrica no neoplásica. }\end{array}$} \\
\hline DIAGNOSTICO & $\begin{array}{l}H P(t) \\
n(\%)\end{array}$ & $\begin{array}{l}H P(\cdot) \\
n(\%)\end{array}$ & TOTAL & P \\
\hline Ulcera gástrica & $7(100.0)$ & $0(0.00)$ & 7 & \multirow[t]{2}{*}{$p<0.04$} \\
\hline Várices esofágicas & $1(100.0)$ & $0(0.00)$ & 1 & \\
\hline Ulcera duodenal & $9(81.82)$ & $2(18.18)$ & 11 & \multirow[t]{7}{*}{$p<0.094$} \\
\hline Gastritis" & $105(81.40)$ & $24(18.60)$ & 129 & \\
\hline Cicatriz de úlcera" & $4(80.00)$ & $1(20.00)$ & 5 & \\
\hline Pólipos"** & $3(75.00)$ & $1(25.00)$ & 4 & \\
\hline Esofagitis & $14(58.33)$ & $10(41.67)$ & 24 & \\
\hline NORMAL & $1(50.00)$ & $1(50.00)$ & 2 & \\
\hline TOTAL & $144(78.69)$ & $39(21.31)$ & 183 & \\
\hline 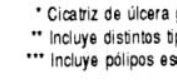 & $\begin{array}{l}\text { vodenal. } \\
\text { picios de gastit } \\
\text { stricos y guode }\end{array}$ & & & \\
\hline
\end{tabular}

Relación entre diagnóstico endoscópico y el hallazgo histológico de gastritis en la mucosa gástrica no neoplásica. 
Hay una alta frecuencia del hallazgo histológico de gastritis en presencia de otros diagnósticos de patología endoscópica; en contraste con la presencia de esta alteración histológica en el $100 \%$ de los casos que el endoscopista consideraba mucosa normal. Los resultados se encuentran en la tabla $\mathrm{N}^{\circ} 7$ y tabla $\mathrm{N}^{\circ} 8$.

Tabla $\mathrm{N}^{\circ} 7$. Diagnóstico endoscópico y hallazgo histológico de gastritis en la mucosa gástrica no neoplásica.

\begin{tabular}{lccr} 
DIAGNOSTICO & $\begin{array}{c}\text { GASTRITIS } \\
\text { ENDOSCOPICO }\end{array}$ & $\begin{array}{c}\text { NORMAL } \\
n(\%)\end{array}$ & TOTAL \\
\hline Gastritis* & $125(96.90)$ & $4(3.10)$ & 129 \\
Otra patología & $46(88.46)$ & $6(11.54)$ & 52 \\
NORMAL & $2(100.0)$ & $0(0.00)$ & 2 \\
TOTAL & $173(94.54)$ & $10(2.53)$ & 183
\end{tabular}

* Induye todos los tipos endoscópicos de gastritis. 


\begin{tabular}{|lcc|}
\hline $\begin{array}{c}\text { Tabla N 8. Frecuencia de } \\
\text { en biopsias gástricas según series. }\end{array}$ \\
\hline AUTOR (Referencia) & $n$ & $\%$ \\
\hline Llerena Y. (17) & 295 & 60 \\
Hernández F. y Col. (38) & 92 & 67 \\
Torres E. (5) & 100 & 68 \\
Solari M. y Col. (39) & 100 & 69 \\
Carneiro G. y Col. (48) & 56 & 69.6 \\
Miranda R. (18) & 336 & 73 \\
Ramirez y Col. (31) & 143 & 73 \\
Solari y Col. (59) & 151 & 73.6 \\
Raws E. y Col. (32) & 303 & 77 \\
Gilman R. y Col (36) & 103 & 77.3 \\
Coelho L. y Col (33) & 51 & 78 \\
Presente estudio & 183 & 78.69 \\
Cabello J. y Col (40) & 145 & 80 \\
Mello E. y Col (34) & 153 & 83 \\
Ramirez y Col (29) & 2011 & 84 \\
Solari M. y Col (41) & 190 & 94 \\
\hline
\end{tabular}

\section{DISCUSIÓN}

El uso de los panedoscopios de fibra y actualmente los videoendoscopios en el diagnósticos de las enfermedades del tracto digestivo superior ha dado indiscutibles avances; en la actualidad la endoscopía alta es el examen auxiliar de elección en relación a la radiología en el estudio del esófago, estómago y duodeno, por la gran mayoría de especialistas, por su demostrada sensibilidad y especificidad comparado con los métodos radiológicos, alcanzando tasas de positividad diagnóstica entre el $85 \%$ a $98 \%(7,92,50)$; además porque en comparación con aquellos es de menor costo, por lo que está al alcance de mayor cantidad de pacientes (15). Por otro lado, cabe resaltar el gran avance en el uso terapéutico en las lesiones esófago-gastro-duodenales, como la esclerosis y ligadura de várices esofágicas, con técnicas sencillas y fármacos baratos; la extracción endoscópica de pólipos (1), inyección de sustancias, entre ellas la epinefrina, para detener el sangrado de úlcera pépticas con un gran porcentaje de efectividad, y muchos otros procedimientos que sería largo enumerar.

La patología del tracto digestivo superior es muy amplia; una de las molestias más frecuentes, por las que acuden los pacientes a los servicios de gastroenterología es la dispepsia; ésta llega a porcentajes muy elevados como lo demuestra Barrós en una población aparentemente sana (4), dicha molestia en la actualidad tiene una definición 
imprecisa y se refiere a un grupo de síntomas del tracto digestivo alto y que puede ser manifestación de organicidad o no $(2,3,5)$. Otra patología muy frecuente es la gastritis crónica y que puede se tan alta como el $68 \%$ de las endoscopías realizadas y como el 97\% de los reportes de histología; algunos autores proponen que ésta patología es resultado del envejecimiento de la mucosa gástrica $(7,16,17,18,20)$. Otra patología frecuente por la que acuden los pacientes a los servicios especializados es la enfermedad úlcero péptica; la mayoría de los autores coinciden que su prevalencia es alrededor del 10\% (6-14) y la edad más frecuente de presentación de la úlcera duodenal es de $30-50$ años, mientras que la gástrica es de 40 - 60 años y que existen diversos factores asociados como el uso de ácido acetil salicílico, anti inflamatorio no esteroideos, estados hiper-secretores y últimamente se considera como factor injuriante al Helicobacter pylori $(6,15)$.

El cáncer gástrico es una enfermedad potencialmente letal, por eso la importancia de un diagnóstico oportuno en fases tempranas de la enfermedad; ésta es una patología de pacientes de edad avanzada, pues la literatura reporta una edad avanzada, pues la literatura reporta una edad promedio de 63 años; se encuentra relacionado con la distribución geográfica, llegando a frecuencias tan altas como 80 por cada cien mil habitantes mayores de 45 años en el Japón; en nuestro país es de 5 al 8 por cada cien mil habitantes de la población general $(21,22,23,27,55)$.

En el presente trabajo se ha realizado el estudio retrospectivo de diversos diagnósticos endoscopios y los hallazgos histológicos de las muestras de la mucosa gástrica biopsiadas endoscópicamente, en los pacientes que acudieron al centro de endoscopía alta de la Clínica Médica Cayetano Heredia, con la intención de determinar las características reportadas en dicha población.

El promedio de edad estuvo alrededor de los 45 años; esta edad está alrededor de la mayoría de estudios endoscopios realizados en el país $(12,51)$; esto nos hablaría de un promedio de adultos que acude a este servicio por diversas molestias digestivas. Tampoco hay diferencias entre el sexo de los pacientes; esto se explica porque en CLIMA acuden pacientes de ambos sexos, a diferencia de otros centros hospitalarios en donde hay un claro predominio de atención de pacientes de un sexo respecto al otro $(13,26)$.

Dentro de los diagnósticos endoscopios obtenidos vemos que predomina el de gastritis, esto ha sido reportado por diversos autores con porcentajes que van desde $36 \%$ al $68 \%$ de toda la patología endoscópica alta $(7,8,11,12,13,14,19)$. En lo referente al diagnóstico endoscópico de normalidad, la literatura revisada reporta porcentajes muy variados que van desde el 4.18\% hasta el 40.5\% (7,10,11,12,14,51); hallazgo está dentro del rango encontrado por dichos autores. En lo que respecta al diagnóstico de úlcera gástrica y úlcera duodenal, la frecuencia encontrada es levemente menor que en los trabajos revisados, los cuales van de $3.46 \%$ hasta $15.6 \%$ para la úlcera gástrica y de $4.5 \%$ hasta $26.62 \%$ para la úlcera duodenal $(6,8,11,12,13,19,24,26)$. Se ha hallado en el presente estudio que las úlceras duodenales son más frecuentes que las gástricas en una relación de 1.5; lo encontrado en la literatura mundial va de $10 / 1$ a $3 / 1(6,13,14)$. La frecuencia del hallazgo de lesiones polipoideas es similar a lo ya publicado; en dichos trabajos los porcentajes varían de $1.2 \%$ hasta $4.05 \%(9,10,14)$. Finalmente, la frecuencia del diagnóstico endoscópico de cáncer gástrico, está dentro de lo reportado en la literatura, la que va de 
1.6\% hasta $14.28 \%(15,22-26)$; como lo reporta Nago (27), en el Perú ésta es una patología de gran importancia, pues su frecuencia está alrededor de 8 por cada cien mil habitantes de la población general; siendo en los varones la primera causa de mortalidad debida a neoplasias en la mujer la segunda causa, sólo superada por el cáncer de cuello uterino; la sobreviva de estos pacientes con cáncer avanzado es alrededor del $12 \%$ a los 5 años y depende del estadio, localización y tipo histológico $(21,22)$.

Se hizo biopsias endoscópicas casi a la mitad de los pacientes a los que se le realizó la endoscopía digestiva alta, y la mayor proporción de biopsias se realizó cuando el diagnóstico endoscópico fue de neoplasia, en donde se hizo a la totalidad de los pacientes, seguida por la úlcera gástrica para verificar la naturaleza de esta lesión; hay diversas publicaciones que recomiendan la realización rutinaria de biopsias gástricas como complemento de la endoscopía digestiva alta; el argumento es que no hay una correlación entre el hallazgo macroscópico y microscópico $(52,53,55)$. Además interesa, para muchos casos, determinar la severidad y el tipo de gastritis y la presencia de Helicobacter pylori, con la finalidad de precisar posteriormente el efecto terapéutico de diversos esquemas que en la actualidad se están probando para erradicar el Helicobacter pylori y su consecuente efecto sobre la mucosa gástrica.

En el presente trabajo, se tomaron en cuenta solamente los reportes histológicos de mucosa gástrica no neoplásica, por se ésta la patología más frecuente y porque en el servicio de Inmunopatología del Hospital Nacional Cayetano Heredia en los casos de neoplasia gástrica no se analizan ni reportan los otros cambios que pudieran existir en la mucosa neoplasias requiere de un seguimiento en la evolución de la enfermedad, lo que lamentablemente no se pudo realizar en este estudio.

Los informes de patología nos dan una frecuencia de Helicobacter pylori (Figura $\mathrm{N}^{\circ}$ 7)en mucosa gástrica no neoplásica de $78.69 \%$; dicho resultado están en el promedio de lo reportado en la literatura nacional y extranjera que hemos revisado, los que se encuentran entre $60 \%$ y $94 \%(5,17,18,29,31,32,33,34,38,39,40,41,48,56,59)$, como vemos en la tabla $\mathrm{N}^{\mathrm{o}}$; ; en este estudio se utilizó en todas las muestras la coloración de HE para el diagnóstico del Helicobacter pylori; ésta, como se demuestra en diversos estudios, tiene gran efectividad y es superada sólo por las coloraciones que usan plata (Warthin Starry)(29,56). Los hallazgos histológicos de gastritis, actividad de gastritis, daño mucinoso y metaplasia, también corresponden con lo reportado en la literatura $(5,17,18)$. Hay que resaltar la sistematización en el diagnóstico histológico que se emplea en el servicio de patología del $\mathrm{HNCH}$, ya que por su modo descriptivo permite tener una idea clara de la microscopía de la mucosa gástrica, grados de inflamación, etapa evolutiva de la enfermedad, presencia y grado de infección por Helicobacter pylori, etc., $\mathrm{y}$ es de mucha utilidad para hacer investigación.

Al igual que lo reporta Ramírez Ramos (29), en este estudio no hay diferencia entre la presencia de Helicobacter pylori en la mucosa gástrica no neoplásica con el sexo de los pacientes y con la edad de los mismos, probablemente por la gran tasa de infección de esta bacteria desde edades tempranas de la vida y que contrasta con los reportes de países desarrollados en donde la prevalencia se incrementa con la edad; además está reportado en nuestro país que no existe una correlación entre la presencia del Helicoacter pylori y estrato 
socioeconómico, excepto en mujeres de estrato socioeconómico alto, lo cual está en contraste con literatura norteamericana $(29,30,31,35,36,37)$.

En este estudio se ha encontrado una relación estadísticamente significativa entre el hallazgo de gastritis, sea de cualquier tipo y la presencia de Helicobacter pylori y cuando estudiamos la actividad de la misma y el daño mucinoso, la relación fue aún más significativa; esto ha sido encontrado por diversos autores desde los primeros repotes de Marshall y Warren $(5,17,28,32,33,34,40,47,48)$; los mismos autores también reportan que con la erradicación de la bacteria hay una regresión dramática del grado de inflamación de la mucosa gástrica. El Helicobacter pylori es un organismo patógeno y que debe erradicarse buscando en cada área geográfica el mejor esquema antibiótico. Hasta el momento en nuestro país se observa una tasa alta de reinfección. Otro hallazgo encontrado en este trabajo es la escasa presencia de Helicobacter pylori en mucosas gástricas histológicamente normales, coincidiendo con lo reportado anteriormente por diversos autores $(29,47,57)$.

La relación entre el diagnóstico de úlcera gástrica con la presencia de Helicobacter pylori fue estadísticamente significativa, pero no así con la úlcera duodenal; esto no corresponde con lo descrito por diversidad de autores, pues ellos encuentran mayor relación con la úlcera duodenal, esto requiere de un estudio posterior para determina las causas de este hallazgo; una posibilidad es que algunas de estas úlceras duodenales hayan estado en relación al uso de anti inflamatorios no esteroideos $(38,47,48)$; también se propone que en estas patologías se debe dar tratamiento antibiótico, para erradicar a dicho bacilo; pues existe trabajos que demuestran la gran disminución en la tasa de recurrencia y resangrado de las úlceras pépticas, sean gástricas o duodenales, con estas medidas terapéuticas $(30,42-$ 45). Los esquemas antibióticos para la erradicación del Helicobacter pylori son muy variados, pero todos coinciden en que se debe usar terapia combinada debido a la gran capacidad de generar resistencia antibiótica por la bacteria y por la baja tasa de erradicación con monoterapia (30.37.43). Ante estas evidencias, hay la necesidad de dar a nuestros pacientes con cualquier tipo de úlcera, la terapia de erradicación del Helicobacter pylori, educar a los pacientes sobre los riesgos y beneficios del tratamiento y también la educación al personal médico empezando desde el pregrado. En lo que respecta al tratamiento a emplear, hay muchos esquemas; pero por las características económicas de los paciente hay que optar por regímenes no tan costosos para asegura el cumplimento del tratamiento y así evita resistencia bacteriana. Finalmente, cabe resaltar, que no existe una lesión endoscópica patognomónica en la mucosa gástrica ocasionada por el Helicobacter pylori (58).

Se encontró una alta frecuencia de la presencia histológica de gastritis coincidente con otras patologías diagnosticadas endoscópicamente, por el contrario los resultados que obtuvimos al comparar el diagnóstico endoscópico de normalidad y el diagnóstico histológico nos muestra la falta de relación entre ambas, esto ha sido descrito anteriormente por varios autores nacionales y extranjeros $(52,53,54)$. Este hallazgo nos llevaría a sugerir la necesidad de realizar biopsias en los procedimientos endoscopios; pues la endoscopía sola no nos puede dar datos sobre el tipo de gastritis, su actividad o la presencia de Helicobacter pylori.

Como corolario de este trabajo, se sugiere la conveniencia de estandarizar la terminología empleada en los reportes de los diagnósticos endoscopios, para uniformizar los diferentes 
criterios empleados en la denominación de la variada patología del tracto digestivo superior. Además la falta de relación entre el diagnóstico endoscópico de normalidad y el hallazgo histológico, nos lleva a sugerir la necesidad, de se posible, de realizar las biopsias endoscópicas de manera rutinaria. Finalmente convendría estudiar todos los cambios que se producen en la mucosa gástrica, en las diversas patologías incluyendo la neoplasia de estómago.

\section{REFERENCIAS BIBLIOGRAFICAS}

1.Cantella M. Polipectomía endoscópica en el tracto digestivo alto. Tesis de Bachiller en Medicina. Lima, Perú. Universidad Peruana Cayetano Heredia, 1982.

2.Peredea V. Redefiniendo dispepsia. Rev Gastroent Perú 1992; 12:28-34.

3.Mc Quaid K. Symptoms and signs of gastrointestinal disease. Current of Medical Diagnosis and Treatment 1996: 489-490.

4.Barrós, P. Molestias digestivas en la población adulta aparentemente sana en Lima metropolitana según sexo, edad y nivel socioeconómico. Tesis de Bachiller en Medicina. Lima, Perú. Universidad Peruana Cayetano Heredia, 1989.

5.Torres E. Hallazgos clínicos, endoscopios e histológicos en pacientes dispépticos. Tesis para Optar el Título de Médico Cirujano. Lima, Perú. Universidad Peruana Cayetano Heredia, 1996.

6.Espejo Romero H. Enfermedad ulcerosa.En: IPSS. Manual de Gastroenterología IPSS. 1995: 36-68.

7.Lanao F, Farfán G, Bussalleu A, Berríos J, Pineda R. Endoscopía digestiva alta en el Hospital Nacional Cayetano Heredia. Correlación con otros métodos diagnósticos. Rev Gastroent Perú 1986; 6:134-142.

8.Praelli J, Morales C. Endoscopía digestiva alta en el Hospital Nacional Dos de Mayo. Lima, Perú: VI Congreso Peruano de Gastroenterología; 1978:36.

9.Barrenzuela L, Lozano R, Junchaya V, Serrano H. Endoscopia Per Oral: 1978-1981. VIII Congreso Peruano de Gastroenterología; 1982:29.

10.Farfán G, Berríos J, Huamán C, Olivares A. Estudio de 441 exámenes del tracto digestivo superior. VIII Congreso Peruano de Gastroenterología 1982:30.

11.Racchumí M, Delgado G. Endoscopía Alta en el Hospital Central del Norte. IX Congreso Peruano de Gastroenterología. Lima 1984: 118.

12.Olivares A, Chávez J, Rodríguez J. Endoscopía digestiva alta en el Hospital de PetroPerú de Talara. XI Congreso Peruano de Gastroenterología. Lima 1988: 136.

13.Nago A, Peña L. Resultado de 30,646 esófago gastroduodenoscopías (25-9-79 a 30-1290) Hospital Arzobispo Loayza. Trujillo: XII Congreso Peruano de Enfermedades Digestivas; 1990: 84.

14.Racchumí M, Delgado G, Ruiz M, Ruiz O. Endoscopía Digestiva Alta en el Hospital Regional Central del Norte. Trujillo: XII Congreso Peruano de Enfermedades Digestivas; 1990: 82.

15.Berríos J, Tataje J, Bussalleu A, Farfán G, Combe J, Pineda R. Ulcera péptica en el Hospital Cayetano Heredia. Lima: X Congreso Peruano de Gastroenterología; 1986: 54.

16.Espejo Romero H. Gastritis Crónica. En: IPSS Manual de Gastroenterología. IPSS; 1995: 30-35. 
17.Llerena Y. Biopsias gástricas endoscopías no neoplásicas en el Hospital de Apoyo N² Cayetano Heredia (Período 1981-1985). Tesis para Optar el Título de Especialista en Patología y Laboratorio Clínico. Lima, Perú. Universidad Peruana Cayetano Heredia, 1991. 18.Miranda R. Revisión histopatológica de las biopsias gástricas endoscópicas no neoplásicas, Período 1986 - 1987, en el Hospital de Apoyo N² Cayetano Heredia. Tesis para Optar el Título de Especialista en Patología y Laboratorio Clínico. Lima, Perú. Universidad Peruana Cayetano Heredia, 1988.

19.Sánchez V. Fibroendoscopía en la Ciudad de Ica, resultado de 470 exámenes. Lima: VI Congreso Peruano de Gastroenterología; 1978: 38.

20.Spiro H. Enfermedades inflamatorias. En:Gastroenterología clínica. 3era. Edición. 1986 :243-277.

21.Spiro H. Cáncer gástrico. En: Gastroenterología Clínica. 3era edición 1986: 291-311.

22.Espejo H. Cáncer gástrico. Rev Gastroent Perú 1991; 11: 48-61.

23.Destefano V. Carcinoma Gástrico: Estudio clínico patológico Centro Naval del Perú 1979 -1989. Tesis de Bachiller en Medicina. Lima, Perú. Universidad Peruana Cayetano Heredia, 1995.

24.Candela R, Pineda, Gálvez G, et al. Cáncer Gástrico. Estudio Multicéntrico en Seis Hospitales de Lima y Callao. Rev Gastroent Perú 1986; 6: 92-96.

25.Ayala G, Cisneros G. Hidalgo G. Diagnóstico Endoscópico del Carcinoma Gástrico Hospital Eugenio Espejo, Quito. Quito: XX Congreso Panamericano de Gastroenterología. 1988: 106.

26.Celestino A, Espejo H. Aspectos Clínicos del Cáncer Gástrico en el Hospital Central del Empleado. Lima :III Congreso Peruano de Gastroenterología;1972: 91.

27.Nago A, Jiménez R, Verdi G. Cáncer Gástrico como Problema de Salud en el Perú. Lima: IX Congreso Peruano de Gastroenterología; 1984: 45.

28.Warren J, Marshall B. Undertified curved bacillus in gastric epitelium in active chronic gastritis. Lancet 1983; 1: 1273-1275.

29.Ramírez A, Gilman R, Recavarren S, et al. Algunas características de la infección por Helicobacter pylori en el Perú. Acta Gastroent Latin Americ 1991; 21(4): 211-219.

30.Peterson W. Helicobacter pylori and peptic ulcer disease. N Engl J Med 191; 324(15): 1043-1048.

31.Ramírez A, Hurtado O, Rodríguez C, Garrido G, León R, et al. Campylobacter pilórico y nivel socioeconómico. Acta Gastroent Latin Americ 1987; 17: 35-42.

32.Raws E, Langenberg W, Houthoff H, Zanen H, Tytgat G. Campylobacter pyloridis associated chronic active antral gastritis. Gastroenterology 1988; 94: 33-40.

33.Coelho L, Das S, Karim Q, et al. Campylobacter pyloridis in the upper gastrointestinal tract: a brazilian study. Arq Gastroenterol Sao Paulo 1987; 24 (1): 5-9.

34.Mello E, Melo C. Prevalencia dos diferentes tipos de gastrites em pacientes com quexias altas. Arq Gastroenterol Sao Paulo 1991; 29(29): 43-50.

35.Ramírez A, León R, Gilman R, Recavarren G, Berendson R, et al. Helicobacter pylori and gastritis in peruvian patients: relationship to socioeconomic level, age and sex. Am $\mathrm{J}$ Gastroenterol 1990; 85(7): 819-823.

36.Ramírez A, Gilman R, Spira W, et al. Ecology of Helicobacter pylori in Perú: infection rates in coastal, high altitude, and jungle communities. Gut 1992; 33: 98-99.

37.Ramírez Ramos A., Helicobacter pylori. Rev Gastroent Perú 1991; 11: 32-38.

38.Hernández F, Rivera $\mathrm{P}$, et al.. The first cases of Helicobacter pylori (Campylobacter pylori) reported from Costa Rica. Rev Biol Trop 1990; 38(2B): 481-482. 
39.Solari M, Garay A, Candela R, et al. Experiencia en 100 casos de Gastritis Crónica y Ulcera Gástrica Asociado a Campylobacter pilórico. XI Congreso Peruano de Gastroenterología. Lima 1988: 18.

40.Cabello J, Berríos J, Bussalleu A, et al. Gastritis, ulcera gástrica y campylobacter pylori. Lima: XI Congreso Peruano de Gastroenterología; 1988: 14.

41.Solari M, Candela R, Rebaza S, et al. Identificación del Helicobacter pylori con Coloración Maysson y HE en Asintomáticos y Sintomáticos. Lima: XIII Congreso Peruano de Enfermedades Digestivas; 1992: 10.

42.Rauws E, Tytgat G. Cure of duodenal ulcer associated with eradication of Helicobacter pylori. Lancet 1990; 335: 1233-1235.

43.Ramírez Ramos A. Tratamiento de la infección por Helicobacter pylori: estado actual. Rev Gastroent Perú 1995; 15 Suplemento (1): S7-S21.

44.Jaspeasen D, Koerner T, Schorr W, Brennenstuhl M, Raschka C, Hammar C. Helicobacter pylori eradication reduces the rate of rebleeding in ulcer hemorrhage. Gastrointestinal Endoscopy 1995; 41(1): 5-7.

45.Rokkas T, Karameris A, Mavrogeorgis A, Rallis E, Giannikos N. Eradication of Helicobacter pylori reduces the possibility of rebleeding in peptic ulcer disease. Gastrointestinal Endoscopy 1995; 41(1): 1-4.

46.Owen D. Stomach. Histology for pathologists 1992: 533-544.

47.Ramírez A, Recavarren S, León R, et al. Campylobacter pilórico, gastritis crónica, úlcera gástrica y úlcera duodenal. Arq Gastroenterol Sao Paulo. 1987; 24(1): 10-15.

48.Carneiro G, Magalhaes M, Galvao L, et al. Ocurrencia do Campylobacter pylori em pacientes com gastrite e ulcera peptica. Arq Gastroenterol Sao Paulo 1988; 25(1): 23-28.

49.Pineda R, Berrios J, Farfán G, et al. Cáncer gástrico en el Hospital Cayetano Heredia 1969-1985. Lima: X Congreso Peruano de Gastroenterología; 1986: 70.

50.Ramos R, Hernández J, Bussalleu A. Cáncer gástrico en el Hospital Cayetano Heredia. Revisión Epidemiológica, Clínica y Anatomopatológica. Lima: XI Congreso Peruano de Gastroenterología; 1988: 32.

51.Lanao F, Farfán G, Bussalleu A, Berríos J. Endoscopías digestivas altas en el Servicio de Gastroenterología del Hospital de Apoyo Cayetano Heredia. (1975-1985). Lima: X Congreso Peruano de Gastroenterología; 1986: 49.

52.Carpenter H, Talley N. Gastroscopy is complete Gastropathy From Gastritis. Gastroenterology 1995; 108: 917-924.

53.Ramírez A, Watanabe J, Recavarren S, Cok J, Zevallos E, Hurtado O. Correlación entre el diagnóstico endoscópico e histológico en la gastritis crónica astral. Rev Gastroent Perú 1986; 6: 171-177.

54.Polit R, López A. Estudio comparativo entre los aspectos endoscópicos e histopatológicos en la gastritis crónica atrófica. Quito: XX Congreso Panamericano de Gastroenterología; 1988: 48.

55.Ramírez A, Galarza J, Barreda C, et al. Importancia del Seguimiento de la Ulcera Gástrica. Rev Gastroent Perú 1982; 2: 15-19.

56.Gilman R, Recavarren S, Spira W, et al. Comparación de métodos para detectar el Campylobacter pilórico. Lima: X Congreso Peruano de Gastroenterología. 1986: 1.

57.Ramírez Ramos A., Recavarren S., León Barúa R,. Garrido G., Gilman R., Spira W., Watanabe J., Cok J., Cevallos E. Campylobacter pilórico en mucosa gástrica astral normal Rev Gastroent Perú 1986; 6: 125-126. 
58.Olgy S, Mendoza S, Gerlud M. Frecuencia del Campilobacter pylori en las Lesiones Gastroduodenales en la Consulta Gastroenterológica. GEN 1988; 42: 174-176.

59.Solari M, Candela R, Saettone L, et al. Gastritis Crónica Activa y Campylobacter Pylori. Trujillo: XII Congreso Peruano de Enfermedades Digestivas. 1990:14.

Rev Med Hered 1997; 8: 58-66 